\title{
Radiographic Study of the Correction of Radial Length in Wrist Fracture: The Ulnar Variance Compared With the Controlateral Side in 95 Cases Treated With Intra Focal Pinning
}

\author{
Hassan Najdi' ${ }^{*}$, Dany Mouarbes ${ }^{2}$, Fady Jammoul ${ }^{2}$, Ali Ibrahim² ${ }^{2}$, Ali Chamseddine ${ }^{3}$, Roger Jawish ${ }^{4}$ \\ ${ }^{1}$ Orthopaedic Surgery department, Sacré-Coeur Hospital, Hazmieh Lebanon
}

${ }^{2}$ Head of Orthopaedics \& Trauma Surgery at the Faculty of Medical Sciences, Lebanese University, Hazmieh Lebanon

${ }^{3}$ Head Department of Orthopedic Surgery, Sacré-Coeur Hospital, Hazmieh Lebanon

Received: February 05, 2018; Published: February 16, 2018

*Corresponding author: Hassan Najdi, Orthopedic Surgery department, Sacré-Coeur, Hazmieh Lebanon, Middle East, Tel: 9613 852012; Email: najdihassan@hotmail.com

\begin{abstract}
Introduction: Values of different parameters for correction of distal radius fracture is well defined, except for ulnar variance (UV) which demonstrates variant morphologic values between individuals.

Purpose: Correction of radial length in wrist's fracture is more reliable if we consider the UV aspect of the contra-lateral side as reference.

Materials and methods: Prospective study on all patients presented between 2013 and 2016 with extra-articular (Kapandji type1 and 2) and simple intra-articular fractures (Kapandji type4) of wrist and treated by Kapandji technique where contra-lateral UV was used as reference for correction of radial length. Radiographic evaluation of results done 6 months post-operatively.

Results: 95 patients, 61 females, 34 males with mean age 59. In fractured side, the UV was: Neutral (63), Positive (19) and Negative (13). From 65 initial neutral UV, 58 restored the neutral aspect (89\%), 4 became positive (6\%) and 3 became negative (5\%). From 18 initial positive UV, 15 restored the positive aspect (83\%), 3 became neutral (17\%). From 12 negative UV, 10 restored the initial negative aspect (83\%), 2 became neutral (17\%). The contra-lateral UV was: Neutral (65), Positive (18) and Negative (12). Considering the neutral aspect as standard reference for reduction, 63/95 obtained good reduction. Comparing corrected UV to contra-lateral side, 83/95 cases restored the initial aspect and 6 cases were over lengthened.
\end{abstract}

Conclusion: Considering the contra-lateral UV as reference for correction, the normalization rate of UV is $87.3 \%$ instead of $66.3 \%$ when neutral aspect of UV is considered as standard anatomy.

Keywords: Wrist Fracture; Intra Focal Pinning; Ulnar Variance; Radial Length.

\section{Introduction}

Distal radius fractures are one of the most common types of fractures, it accounts up to $25 \%$ of fractures in children [1] and up to $18 \%$ of fractures in the over 65 age group $[2,3,4]$.The treatment depend on several factors of stability: the radial length, the metaphysical comminution and the age of patients. But, whatever is the type of treatment (conservative or surgical), it relies on standard values of palmar tilt, radial inclination and ulnar variance to evaluate the good correction of fracture. While palmar tilt and radial inclination have well defined values, the UV miss a specific value to lay on for correction. Three aspects of UV are known: neutral, positive and negative, with different incidences in normal population where the neutral aspect is the most frequent and therefore it is considered as the standard aspect of assessment during surgery. This UV is also considered as the most consistent predictor of radiographic outcome between the other parameters of reduction (palmar tilt and radial inclination) [5-9], therefore the relevance of procedures of reduction, except the internal fixation, remained uncertain due to the imprecision to restore the initial 
aspect of UV by considering the neutral aspect as standard of assessment without taking into consideration the variation in the morphology of wrist that exists between individuals.

We have assessed the reduction of distal radius fractures in a prospective series of patients treated with trans-focal pinning of the Kapandji technique [10-12] for comminuted extra articular (type 1 and 2) or extra-articular with simple intra-articular fractures (type 4) using UV of the contra lateral side as reference to restore the radial length. The aim of our study was to demonstrate that the standard value of the radial length after reductionis obviously submitted to individual pattern and should be provided by the ulnar variance of the contra lateral wrist. The trans-focal pinning of the Kapandji technique is known to have lesser effect of lengthening of the radius than other methods, without evidence for this statement. This notification could be sustained when we consider wrongly the neutral UV type as the standard aspect for all individuals. However, in this study, we assess the length of the radius performing the mentioned technique, according to the UV at the opposite wrist. The result changes if we consider the neutral UV for all patients or the individual pattern verified at the opposite normal side.

\section{Materials and Methods}

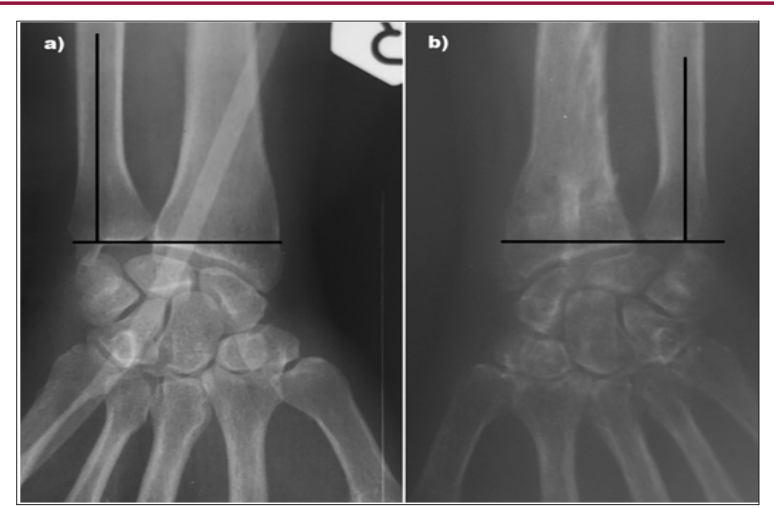

Figure 1: Wrist $x$-ray (AP) Showing the neutral aspect of ulnar variance in the non-fractured side (a), As well as in the operated side after removal of pins (b).

Prospective study on all patients presented between 2013 and 2016 with extra-articular (Kapandji type1 and 2) and simple intraarticular fractures (Kapandji type 4) of wrist. All patients presented to our institution with wrist trauma underwent an X-ray (AP and LAT) of the painful wrist. The patients that their X-rays showed an extra-articular (Kapandji type1 and 2) and simple intra-articular fractures (Kapandji type 4) of wrist and candidates for treatment with Kapandji technique were included in the study, and an X-ray (AP and LAT) of the contra lateral wrist was performed after permission of the patients. Open fractures, fracture of distal radius associated with distal ulnar fracture were excluded, as well as patients whose we didn't obtain an X-ray of opposite non-fractured wrist for different reasons. In each case, two orthopedic surgeons evaluated the UV in the X-ray of contra lateral non-fractured wrist and considered as reference for the restoration of radial length in the fractured side. The UV is considered neutral, when the carpoulnar and carpo-radial surfaces are at the same level, positive when carpo-ulna is projected more distally and negative when carpo-ulna is more proximally (Figures 1-3).

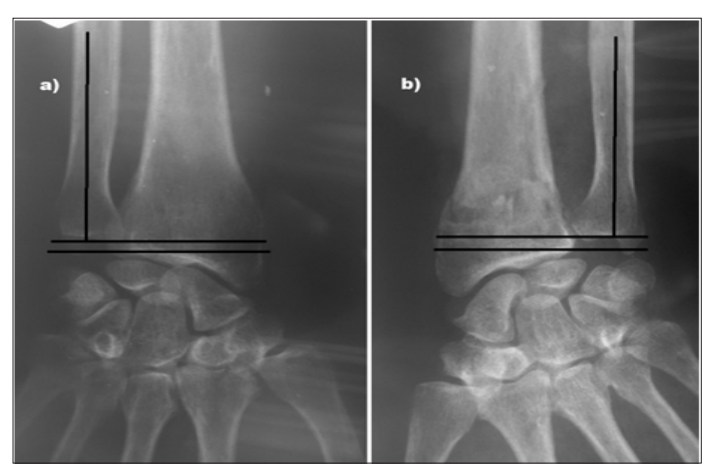

Figure 2: Wrist $x$-ray $(\mathrm{AP})$ showing the negative aspect of ulnar variance in the non-fractured side (a), As well as in the operated side after removal of pins (b).

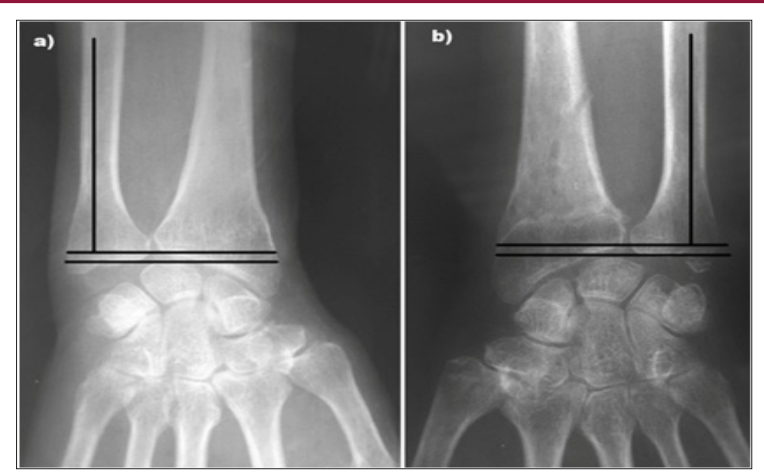

Figure 3: Wrist $x$-ray (AP) showing the positive aspect of ulnar variance in the non-fractured side (a), As well as in the operated side after removal of pins (b).

All patients were treated with the same surgical technique, under general or loco regional anesthesia, in the supine position with the upper limb on a side table, and the use of a tourniquet. After gentle closed reduction the surgeon faithfully followed the surgical technique described by Kapandji [10,13-15], using 3 unthreaded pins $18 / 10$ to $22 / 10 \mathrm{~mm}$ diameter. Fluoroscopic C-Arm control was used to assess reduction and appropriate position of pins at the end of fixation, and the pins were cut back around $1 \mathrm{~cm}$ from the dorsal cortex; they were finally buried between the superficial layer of the dorsal extensor retinaculum and the deep layer of the skin at the time of closure. An above elbow cast was applied for articular fracture and short cast for non-articular fracture. At six weeks (eight weeks for osteoporotic), after a radiographic control, the cast and the 3 intra-focal pins (one lateral and 2 dorsally) were removed and rehabilitation of the wrist was started. A regular follow-up each 2 months was done and at 6 months' post-op an X-ray of control for the treated wrist was done with evaluation of the aspect of UV by two orthopedic surgeons.

\section{Results}

95 patients were treated with the Kapandji technique with at least 6 months postoperative follow-up. 61 females and 34 males between 17 and 85 year olds, mea age 59 years. With 84 cases of extra articularand 11 cases of simple intra-articular fractures 
of the wrist. The UV of the contra lateral side of the 95 cases was neutral in 65 cases, positive in 18 cases and negative in 12 cases. After reduction of the 95 fractures, the UV of the whole group was distributed as followed: Neutral (63 cases), Positive (19 cases) and Negative (13 cases) (Table 1). For the 65 cases of neutral UV in the contra lateral side, 58 cases of treated side restored neutral aspect after reduction (89\%), 4 became positive (6\%) and 3 became negative with over lengthening of the radius (5\%). For the 18 cases of positive UV in the contra lateral side, 15 cases restored the positive UV in the treated side (83\%), 3 became neutral with over lengthening of the radius (17\%). For the 12 cases of negative UV in the contra lateral side, 10 restore a negative variance (83\%) and 2 became neutral variance (17\%) (Table 2). In 83 of 95 cases $(87.37 \%)$, the operated side had the same UV aspect as the contra lateral side, while 6 cases were over lengthened (3 cases in the group of neutral initial variance and 3 cases in the group of positive initial variance). In 6 cases the UV hasn't been restored ( 4 cases of initial neutral and 2 cases of initial negative), because osteoporotic fractures (4 cases), and complex non-indicated articular fracture ( 2 cases). When the neutral UV is considered as the standard reference for reduction of the radial length in all cases, only 63 of 95 (66.31\%) obtain good reduction. In all the series, no cases of serious infection were observed.

Table 1: Postoperative distribution of ulnar variance aspect in operated side as well as in the non-operated side.

\begin{tabular}{|c|l|l|l|}
\hline Ulnar variance & Neutral & Positive & Negative \\
\hline The fractured wrist & 63 cases & 19 cases & 13 cases \\
\hline The non-fractured side & 65 cases & 18 cases & 12 cases \\
\hline
\end{tabular}

Table 2: Results of ulnar variance aspect in the operated side after reduction according to different aspect of ulnar variance in the non-operated side.

\begin{tabular}{|c|c|c|c|}
\hline $\begin{array}{r}\text { Ulnar variance in the } \\
\text { non-fractured side }\end{array}$ & $\begin{array}{c}\text { Neutral: } \\
\mathbf{n = 6 5} \text { cases }\end{array}$ & $\begin{array}{c}\text { Positive: } \\
\mathbf{n = 1 8} \\
\text { cases }\end{array}$ & $\begin{array}{c}\text { Negative: } \\
\mathbf{n = 1 2} \text { cases }\end{array}$ \\
$\begin{array}{c}\text { Ulnar variance After } \\
\text { reduction }\end{array}$ & 58 cases & 3 cases & 2 cases \\
\hline Neutral & 4 cases & 15 cases & - \\
\hline Positive & 3 cases & - & 10 cases \\
\hline Negative & &
\end{tabular}

\section{Discussion}

The most important finding of the present study is that by considering the UV of contra lateral side as reference for correction, we obtained $87.3 \%$ of cases demonstrated a normalization of the radial length, compared to $66.3 \%$ of cases if the neutral UV was considered as reference for correction. Therefore, it is important to take into consideration the variation in the morphology of wrist that exists between individuals, during treatment of wrist fracture. We confirmed our hypothesis: that in absence of standard value for the correction of the radial length, the contra-lateral aspect of the UV could be considered the reference in the treatment of the wrist fracture. This method allows the normalization of the UV according to individual morphology for stabilization of the fractured wrist. It promotes the treatment with pinning which is known to under lengthen the radial fracture. Standard morphologic criteria are required in the treatment of wrist fractures in aim to achieve good outcome. Although, authors overwhelmed their studies with the clinical and radiological assessments and reported results ranging between 92\% [16], 93.75\% [17] and 90.7\% [18], these rates considered the heeling of the fracture and the functional aspect of the wrist. It is obvious that internal fixation with plates provides the restoration for all parameters, whereas pinning has the inconvenience of not restoring always the length of radius, responsible of medial deviation of the wrist

The two parameters, palmar tilt and radial inclination, are determined with a range of standard angles, and accept a range of correction without functional disturbance. However, the UV which is considered the main factor of stability of wrist fracture $[9,11,12,19-21]$ is depending of the carpo-ulnar and the radioulnar articulations, and a minimal insufficient correction promotes a significant clinical deformity. Therefore, Wolfe S. [22] considered that a shortening more than $3 \mathrm{~mm}$ of the ulnar variance will lead to symptoms. Aro HT and Koivunen T [9] have ranged the importance of shortening of the ulnar variance in 2 grades, if the shortening is less or more $5 \mathrm{~mm}$ to promote wrist discomfort. Three distinguished aspects of UV are considered in normal population with variable incidence: neutral, positive and negative. Even though, neutral aspect is the most frequent, it is considered by most authors as the standard parameter for restoration of the radial length [20, $21,23]$. This wrongful consideration is responsible of precarious normalization of fractures, and the UV standard aspect should be considered by the contra lateral side, as individual morphologic reference for correction. Until consolidation of the fracture fixed with pins, changes could occur during the time of consolidation $[9,19]$, therefore different parameters should not be considered postoperatively $[22,24,25]$, but certainly after removal of the pins. Effectively, Brady 0 et al. [24] have reported that between the time of wire removal and final review, there was significant recurrence of dorsal angulations, but no significant radial shortening on radiographs.

The result of restoration of the radial length is not well demonstrated in many of the reports studying the wrist's fractures correction; we expect the reason by lack of standard value. However, Dawdy PA et al. [23] have reported a small series of 17 patients with reference to the non-injured wrist, the UV average for cases was $0 \mathrm{~mm}$ at follow up (average 42 week) v/s $1 \mathrm{~mm}$ average at opposite side, but the authors didn't refer the result to different types of UV. In our study based on the restoration of the initial UV, the normalization in negative (83\%) and positive type (83\%) was lesser than the neutral type (89\%). Considering the whole series, 83 out of $95(87.3 \%)$ cases demonstrated normalization of the radius length. However, if we consider the neutral UV, which represents $68.4 \%$ of our patients, as the standard pattern for our study, the rate of correction falls to 63 out of 95 (66.3\%) cases. Although 
the pinning technique is known to under-correct the length of the radius, in 6 cases the radius was lengthened more than the original ( 3 initial neutrals to negative and 3 initial positive to neutral). The effect of lengthening using the intra-focal pinning in whole patients has consequently a more consideration and reached 89 out of 95 (94\%) cases. In the remaining 6 cases where the technique failed to restore the initial length of radius, we recognize the presence of complex articular fracture and other osteoporotic fractures. In severe osteoporotic, other procedures should be adapted to the weakness of the bone, shortening of the ulna could be an alternative method [26].

\section{References}

1. Ward WT, Rihn JA (2006) The impact of trauma in an urban pediatric orthopedic practice. J Bone Joint Surg Am 88(12): 2759-2764.

2. Baron JA, Karagas M, Barrett J, Kniffin W, Malenka D, et al. (1996) Basic epidemiology of fractures of the upper and lower limb among Americans over 65 years of age. Epidemiology 7(6): 612-618.

3. Kate W Nellans, Evan Kowalski, Kevin C Chung (2012) Epidemiology of distal radius fracture. Hand Clin 28(2): 113-125.

4. BR Singer, GJ McLaughlin, CM Robinson, J Christie (1998) Epidemiology of fractures in 15000 adults; the influence of age and gender JBJS 80 (20): 243-248.

5. Thomas E, Trumble MD, Susan R, Schmitt MD, Nicholas, et al. (1994) Factors affecting functional outcome of displaced intra articular distal radius fractures. The Journal of Hand Surgery 19(2): 325-340.

6. Sumit Batra, Ajay Gupta (2002) The effect of fracture-related factors on the functional outcome at 1 year in distal radius fractures. Injury 33(6): 499-502.

7. Ng CY, McQueen MM (2011) What are the radiological predictors of functional outcome following fractures of the distal radius? J Bone Joint Surg 93(2): 145-150.

8. PJ Mackenney (2006) Prediction of Instability in Distal Radial Fracture J Bone Joint Surg Am 88(9): 1944 -1951.

9. Aro HT, Koivunen T (1991) Minor axial shortening of the radius affects outcome of Colles' fracture treatment. J Hand Surg Am 16(3): 392-398.

10. Kapandji A (1976) L'osteosynthese par double embrochageintrafocal. Traitement fonctionnel des fractures non articulaires de l'extremiteinferieure du radius, Ann Chir 30: 903-908.

11. Weil WM, Trumble TE (2005) Treatment of distal radius fractures with intrafocal (kapandji) pinning and supplemental skeletal stabilization. Hand Clin 21(3): 317-328.

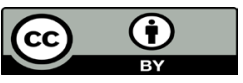

This work is licensed under Creative Commons Attribution 4.0 License

Submission Link: http://biomedres.us/submit-manuscript.php
12. Greatting , Bishop AT (1993) Intrafocal (Kapandji) pinning of unstable fractures of the distal radius. Orthop Clin North Am 24(2): 301-307.

13. Kapandji A (1987) L'embrochage intra focal des fractures de l'extrémité inférieure du radius dix ans après. Ann Chir Main 6(1): 57-63.

14. Hoël G, Kapandji A (1995) Ostéosynthèse par broches intrafocales des fractures à déplacement antérieur de l'épiphyse radiale inférieure. Ann Chir Main 14(3): 142-156.

15. Kapandji A (1998) Ostéosynthèse par brochage intrafocal de type "Arum" des fractures récentes du radius distal chez l'adulte. In: Fractures du radius distal de l'adulte. Cahiers d'enseignement de la SOFCOT. № 67. Sous la direction de Allieu Y. Paris: Expansion Scientifique Publications p. 67-83.

16. Low CK, Liau KH, Chew WY (2001) Results of distal radial fractures treated by intra-focal pin fixation. Ann Acad Med Singapore 30(6): 573576.

17. Das A, Sundaram N, Prasad T, Thanhavelu S (2011) Percutaneous pinning for non-comminuted extra-articular fractures of distal radius, Indian J Orthop 45(5): 422-426.

18. Chin En Chen, Rei JahnJuhn, Jih Yang Ko (2008) Treatment of Distal Radius Fractures with Percutaneous Pinning and Pin-in-plaster, Hand 3(3): 245-250.

19. Nesbitt KS, Failla JM, Les C (2004) Assessment of instability factors in adult distal radius fractures. J Hand Surg Am 29(6): 1128-1138.

20. AfEkenstam J, Hagert C (1985) Anatomical studies on the geometry and stability of the distal radioulnar joint. Scan J. Plast. Reconstr. Surg. Hand Surg 19(1): 17-25.

21. AfEkenstam F (1992) Anatomy of the distal radioulnar joint. Clinic. Orthop 275: 14-18.

22. Wolfe S (2011) Green Operative Hand Surgery, (6 ${ }^{\text {th }}$ edn). Elsevier Churchill Livingstone, London.

23. Dowdy PA, Patterson SD, King GJ, Roth JH, Chess D (1996) Intrafocal (Kapandji) pinning of unstable distal radius fractures: a preliminary report. J Trauma 40(2): 194-198.

24. Brady O, Rice J, Nicholson P, Kelly E, O’Rourke SK (1999) The unstable distal radial fracture one year post Kapandji intrafocal pinning, injury 30(4): 251-255.

25. Solgaard S (1986) Early displacement of distal radius fracture. ActaOrthop Scand 57(3): 229-231.

26. Jawish R, Najdi H, Chamseddine A (2015) Shortening of the distal ulna to correct ulnar variance in osteoporotic wrist fractures treated with intrafocal pining. Eur J OrthopSurgTraumatol 25(6): 1019-1023.

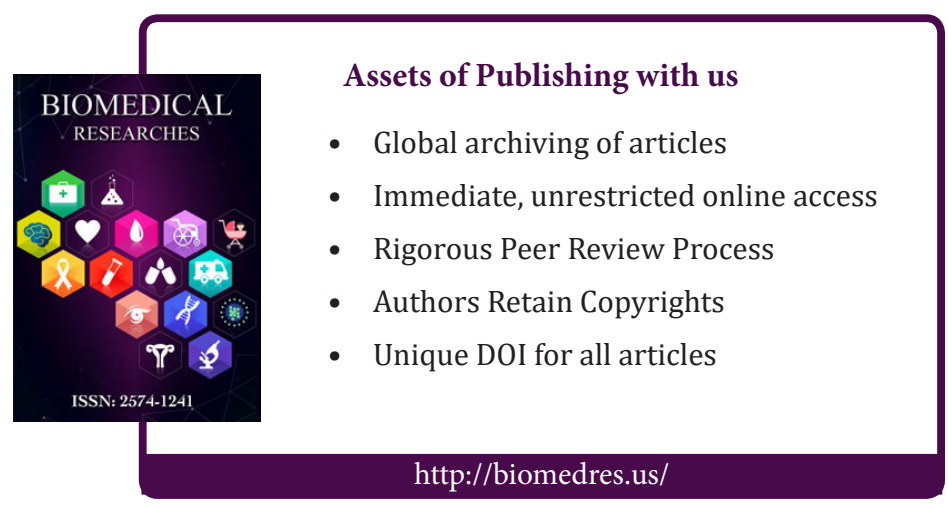

